\title{
POSSIBLE CORRELATION BETWEEN ACUTE ORGANPHOSPHOROUS EXPOSURE AND OCCURRENCE OF SOME CARDIAC ARRHYTHMIA AND BIOCHEMICAL ABNORMALITIES
}

\author{
Mostafa A. Mohamed, Ibrahim A. Abd El-Fattah* and Khaled M. Taha** \\ Department of Forensic Medicine and Clinical Toxicology Faculty of Medicine Al-Azhar \\ University- New Damietta, Cardiology Department Faculty of Medicine Al-Azhar University- \\ Cairo Boys* and General Internal Medicine Faculty of Medicine Mansoura University**
}

\begin{abstract}
Background: Pesticides are often used indiscriminately in large amounts and widespread use of pesticides in agriculture causes environmental pollution and severe health risks such as acute and chronic human poisoning due to acetylcholinesterase enzyme inhibition. A cardiovascular effect of acute organophosphorus (OP) poisoning is very common and requires more estimation. The aim of the work: The present work studied the possible correlation between acute exposure of some (OP) as phosphor dithionate (PDT=Malathion) and diethyl para-nitrophenyl phosphate parathion (DPT=Falidol or Police El-Nagda) and the occurrence of cardiac and biochemical abnormalities. Patients and methods: The study was conducted from the $1^{\text {st }}$ of December 2016 to $1^{\text {st }}$ of October 2017. It involved 100 cases who recruited from the emergency department of Al-Azhar University hospital (New Damietta) and emergency hospital at Mansoura University. In addition to 50 healthy subjects as a control group. After free informed consent to participate in this study and within one hour after admission to general internal medicine, $10 \mathrm{~mL}$ of blood was separated and the serum was divided into three portions, One of which for residues extraction of (PDT) and (DDT)) by using high performance liquid chromatography (HPLC) and the remaining two portions were stored at $-8 \mathrm{C}$ for subsequent assays to all subjects; complete blood count (CBC), fasting blood sugar, serum urea, serum creatinine, serum uric acid, arterial blood gases (ABG), serum electrolytes, cholinesterase level and cardiac enzymes such as (troponin, creatine kinase (CK-MB), lactate dehydrogenase $(\mathrm{LDH})$, aspartate transaminase (AST) and myoglobin (Mb). Cases and controls were subjected to a standardized questionnaire; major variables included in the interview were social and demographic factors, personal habits, and history of bilharziasis, diabetes, alcoholism and occupational exposure to pesticides. Chest X-ray and Electrocardiography (ECG) and clinical examination to all groups were done with a special attention to manifestation of cardiac abnormalities. Results: Significant positive correlation in the study group of pesticide exposures were observed with serum creatinine, arterial blood gases, serum potassium, moderate and severe reduction in cholinesterase level, cardiac enzyme, QTc prolongation, ST elevation and ventricular extrasystole especially among farmers with moderate and severe exposures and living in rural area. Recommendation: It is recommended that; early diagnosis and perfect treatments can save the life of the patients. Careful observation of EEG changes in patients exposed to (OP) compounds is obligatory parallel to the suitable medical treatment as a QTc prolongation and ventricular extrasystole can cause lethal arrhythmia. People who are in touch with pesticides, especially those who spray
\end{abstract}


farmlands and gardens must awareness sufficient protective instruments and consider safety guidelines. Social media awareness and more researches are needed for pesticide compounds and their hazards on the heart and other vital organs.

Key words: Organophosphorus, Cardiac, Biochemical Abnormalities, HPLC.

\section{INTRODUCTION}

Pesticides are chemical or biological substances used to kill or repel targeted organisms (Gilden et al., 2010).

During the past 50 years, pesticides have had an essential role in agricultural and domestic settings throughout the world (International Programme on Chemical Safety and World Health Organization, 2014).

Easy availability of the (OP) insecticides has resulted in a gradual increase in occupational, accidental or suicidal poisoning mainly in developing countries (Aardema et al., 2017); (Abdollahi et al., 2017).

Accidental intoxication can occur after acute contact with the skin or inhalation. In addition to spraying, another mode of exposure to pesticides includes formulation and handling without consideration of safety measures. Serious intoxication frequently follows suicidal ingestion (Bhatnagar et al., 2012); (Karki et al., 2014).

(OP) compounds accounts for $80 \%$ of pesticide-related hospital admissions (Linden et al., 2017).

According to the World Health Organization (WHO), one million serious accidentals and two million suicidal poisonings with (OP) occur worldwide every year, and approximately 200,000 died, mostly in developing countries (Sivagnanam, 2014).

The cardiac manifestations occur in a majority of affected patients and may range from harmless electrocardiographic manifestations, such as sinus tachycardia, to lifethreatening complications, such as cardiogenic pulmonary edema (Tafuri and Roberts, 2015).

The mechanisms of cardiac toxicity may be related to sympathetic and parasympathetic over-stimulation, hypoxemia, acidosis, electrolyte imbalances, the direct toxic effect of (OP) compounds on the myocardium and vascular system (Taira et al., 2016).

On the other hand, the use of anticholinergic drugs as an antidote for (OP) intoxication may induce fatal arrhythmias (Worek et al., 2014).

The (OP) compounds induced cardiac intoxication has not been adequately studied. The lack of early diagnosis and treatment of intoxicated patients beside failure of suitable cardiac monitoring may endanger the life of the patients (Laudari, et al., 2017).

\section{AIM OF THE WORK}

The aim of the present work is to study the possible correlation between acute exposure to some (OP) insecticides as phosphor dithionate (PDT=Malathion) and diethyl paranitrophenyl phosphate parathion (DPT $=$ Falidol or Police El-Nagda) and the occurrence of some cardiac and biochemical abnormalities.

\section{PATIENTS \& METHODS}

\section{Study Population}

A case-control study was conducted from the 1st of December 2016 to 1 st of October 2017. After taking and signed informed valid consent to participate in 
this study, one hundred cases were recruited from the emergency department of Al-Azhar University hospital (New Damietta) and emergency hospital at Mansoura University. In addition to 50 healthy subjects as a control group.

Cases with documented past history of atrioventricular arrhythmias, congestive heart failure, rheumatic heart diseases, previous heart block, ischemic heart diseases or preexisting motor/sensory neuropathy were excluded from this study.

Cases and controls were enquired if they had a residence in delta area and dealing with pesticide. They were mentally and physically capable of understanding and completing the questionnaire.

Healthy controls were selected with minimal experience of diabetes, chronic diseases, or alcoholism (Miller et al., 2016).

The questionnaire surveys the following issues:

a) Social and demographic factors as (sex, age, occupation, residence "urban or rural" and residential history).

b) The intention of poisoning.

c) Severity of manifestations of poisoning in patients was categorized into;

-mild poisoning (including anorexia, weakness, restlessness, headache, miosis),

-moderate poisoning (including emesis, increased salivation, increased respiratory secretions, diarrhea, GI upset, diaphoresis, muscle fasciculation, cramping), and

-severe poisoning (pinpoint pupil, pulmonary edema, cyanosis, defecation, urination, confusion, ataxia, seizures, cardiac arrest, and coma).
The elapsed period between exposure and admission to the emergency room.

Personal habits (smoker index i.e. a number of cigarette smoker per day multiplied by a number of smoking years) into (mild up to 200, moderate from 200-400 and severe above 400) and alcohol intake within the last 30 days).

History of diabetes and bilharziasis.

History of occupational exposure or spraying to phosphor dithionate (PDT=Malathion) and diethyl paranitrophenyl phosphate parathion (DPT $=$ Falidol or Police El-Nagda).

Agricultural activities (exposure or working in agriculture, types of crops grown and wearing protective clothes).

Clinical Examination:

Cardiac parameters and vital signs including tachycardia (> 100 bpm), bradycardia $(<60 \mathrm{bpm})$, hypotension $(<$ 90/60 $\mathrm{mmHg}$ ), hypertension (considered as over $140 / 90 \mathrm{mmHg}$ ), temperature and respiratory rates.

Chest X-ray and ECG were carried out to all patients in the general medical ward. ECG changes including prolonged QTc interval, ventricular extrasystole, atrial extrasystole, $\mathrm{T}$ inversion, $\mathrm{ST}$ elevation, ST depression, 1st-degree heart block, 2nd-degree heart block, polymorphic ventricular tachycardia and ventricular fibrillation.

QTc was calculated with Bazett's formula:

$$
\mathrm{QTc}=\frac{\mathrm{QT}}{\sqrt{\mathrm{RR}}}
$$

QTc of over $460 \mathrm{msec}$ or 0.41 second in men and over $470 \mathrm{msec}$ or 0.42 second in women were considered as prolonged (Bazett, 2009).

Pesticide Exposure Assessment

Collection of samples:

Once informed consent was obtained, 10 milliliters of venous blood was collected 
by venipuncture between 6 and 7 am, and it's divided into three portions.

a) One of which was collected in EDTA containing test tubes. phosphor dithionate (PDT=Malathion) and diethyl para-nitrophenyl phosphate $(\mathrm{DPT}=$ parathion $=$ Falidol or Police ElNagda) residues extraction were done by using (HPLC true Quaternary gradients $\quad 250 \quad \mathrm{ml} /$ minute, 3625PSI/250bar. Serial No. PF4250362) (USA) grade hexane and acetone (2:1) according to method of (Verebey et al., 1998).

Hexane $(6 \mathrm{~mL})$ and acetone $(3 \mathrm{~mL})$ were added and the contents were shaken at room temp for $30 \mathrm{~min}$ in a mechanical shaker. The extract was centrifuged for $10 \mathrm{~min}$ at $2000 \mathrm{rpm}$ and the clear top layer of hexane was collected in a clean test tube. Clean up of the samples was done by column chromatography. Elute was collected in a $100 \mathrm{~mL}$ beaker and hexane was evaporated to concentrate the samples. The concentrated residues were dissolved in hexane for further analysis (Eriksson et al., 2014).

b) 2nd part of blood was collected in a tube containing fluoride for fasting blood sugar estimation.

c) The serum was separated from the remaining third blood samples and stored at (-8C). The following assays were performed on all subjects.

- CBC, Blood urea, serum creatinine, serum uric acid, (ABG), serum electrolytes, cholinesterase level; normal value $=1900-3800$ IU/L; It usually declines to $<50 \%$ before symptoms are seen;

- mild poisoning: when the level is $20-50 \%$ of normal value (i.e. level $=380-760 \mathrm{IU} / \mathrm{L}$ ),

- Moderate poisoning: when the level is $10-20 \%$ of normal value (i.e. level $=190-380 \mathrm{IU} / \mathrm{L}$ ),
- $\quad$ severe poisoning: when levels less than $10 \%$ of normal value (i.e. level $<190$ IU/L).

- Cardiac enzymes such as troponin, creatine kinase (CK-MB), lactate dehydrogenase (LDH), aspartate transaminase (AST) and myoglobin $(\mathrm{Mb})$ were measured using kits of Pars Azmoon Company.

\section{STATISTICAL ANALYSIS}

Statistical methods of the results were carried out according to Fisher exact tests (Campbel and Machin, 2009).

The collected data were organized, tabulated and statistically analyzed using SPSS software computer package version 16 (SPSS Inc. Chicago, IL). For quantitative data, all the values were expressed as the mean \pm standard deviation (SD) that the values $\mathrm{P}<0.05$ were considered statistically significant. For comparison between the two groups, the students (t) test was used. For qualitative data, number and percent distribution were calculated and Chisquare test $(\chi 2)$ was used for comparison between two groups. Pearson's correlation coefficient was used to test the correlation between variables. $\mathrm{P}<$ 0.05 was considered to be statistically significant.

\section{RESULTS}

1- Social and Demographic Data:

As regards the demographic data of the studied cases, 70 were males $(70.0 \%)$ and 30 were females $(30 \%)$ in the study group, while in control group 33 males $(66 \%)$ and 17 females $(34.0 \%)$. There was no significant difference in sex distribution between the study and control groups $(\mathrm{P}=0.789)$.

The mean ages of the studied groups were $(25.10 \pm 01 \%)$ in cases group and $(20.05 \pm 01 \%)$ in control group. There 
was no significant difference in age distribution between the studied groups $(\mathrm{P}=0.410)$.

As regards occupation in the study group, 66 cases were farmers $(66.0 \%)$, skilled laborer were 16 cases $(16.0 \%)$, student were 10 cases $(10.0 \%)$, civil and not employed were $(4.0 \%)$ each. While in control group 10 cases not employed $(20.0 \%)$, student were 30 cases $(60.0 \%)$, skilled laborer were 6 cases $(12.0 \%)$, civil employed was 1 case $(2.0 \%)$ and farmers were 3 cases (6.0\%), which is statistically insignificant $(\mathrm{P}=0.620)$.

As regards residence distribution in the study group, urban were 10 cases $(10.0 \%)$ and 90 cases $(90.0 \%)$ in a rural area while in control group 30 cases $(60.0 \%)$ urban and 20 cases $(40.0 \%)$ in rural, which is statistically significant $(\mathrm{P}=0.004)$.

Regarding intention of poisoning in the study group, suicidal cases were 28 (28.0\%), homicidal cases were 7 (7.0\%) and accidental cases were 65 $(65.0 \%)$ while in control group suicidal was 1 case $(2.0 \%)$, homicidal were 3 cases $(6.0 \%)$ and accidental were 3 cases $(6.0 \%)$ which are statistically significant $(\mathrm{P}=0.003)$.

The severity of cases in the study group were mild in 30 cases $(30.0 \%)$, moderate in 60 cases $(60.0 \%)$ and severe in 10 cases $(10.0 \%)$ while in control group mild and moderate severity were 2 cases $(4.0 \%)$ and severe were 3 cases $(6.0 \%)$ which is statistically significant $(\mathrm{P}=0.001)$.

Regarding time elapsed since last intake and admission in the study group; less than 24 hours were 65 cases $(65.0 \%)$, from 1 to three days were 32 cases $(32.0 \%)$ and more than 3 days in 3 cases $(3.0 \%)$ while in the control group, less than 24 hours were 3 cases $(6.0 \%)$ and from 1 to three days were 4 cases $(8.0 \%)$, which is statistically significant $(\mathrm{P}=0.002)$.

Regarding the smoker index in the study group; the number of non-smokers were 15 cases $(15.0 \%)$, mild smokers in 25 cases $(25.0 \%)$, moderate in 24 cases $(24.0 \%)$ and heavy in 36 cases $(36.0 \%)$ while smoker index in the control group, the number of non-smokers were 5 cases $(10.0 \%)$, mild smokers in 7 cases $(14.0 \%)$, moderate in 24 cases $(48.0 \%)$ and heavy in 14 cases $(28.0 \%)$. There is a significant difference as regards smoker index between the study and control groups $(\mathrm{P}=0.001)$.

In the study group, past history of bilharziasis was 25 cases $(25.0 \%)$ and diabetes in 10 cases $(10.0 \%)$ while in control group, past history of bilharziasis was 2 cases $(4.0 \%)$ and diabetes in 3 cases $(6.0 \%)$ There was no significant difference in past history between the study and control groups $(\mathrm{P}>0.05)$.

Agricultural activities in the study group, working or helping in 25 cases $(25.0 \%)$ and both exposures and crops are grown in 30 cases $(30.0 \%)$ while in control group working or helping in 3 cases $(6.0 \%)$ and both exposures and crops are grown in one case $(2.0 \%)$. There was a significant difference regarding agricultural activities between the study and control groups $(\mathrm{P}=0.001)$.

The number of cases wearing protective clothing in the study group was $15(15.0 \%)$ and 2 cases $(4.0 \%)$ in the control group, which is statistically insignificant $(\mathrm{P}=0.200)$. (Table 1$)$.

\section{2- Clinical Data:}

As regarding the clinical manifestation, there was no significant difference between cases and controls regarding systolic and diastolic blood pressure, and temperature $(\mathrm{P}>0.05)$.

As regards the clinical manifestation; the mean respiratory and 
heart rates in the studied groups were $(20.32 \pm 1.06)$ and $(50.97 \pm 5.70)$ respectively. While in the control groups the mean respiratory and heart rates were $(18.39 \pm 1.61)$ and $(62.33 \pm 6.04)$ respectively. There was a statistically significant difference in respiratory and heart rates between the studied groups $(\mathrm{P}=0.013)$ and $(\mathrm{P}=$ 0.012 ) respectively. (Table $2 \mathrm{a}$ ).

As regarding chest $\mathrm{x}$-ray, pulmonary edema was positive in $50.0 \%$ of cases which is statistically significant $(\mathrm{P}=0.002)$.

Regarding ECG abnormalities in the studied groups, QTc prolongation were positive in 50 cases $(50.0 \%)$, ventricular extrasystole were positive in 20 cases $(20.0 \%), 1$ st degree heart block was positive in 8 cases $(8.0 \%)$, inverted $\mathrm{T}$ wave were positive in 5 cases $(5.0 \%)$, atrial extrasystole were positive in 4 cases $(4.0 \%)$, ST elevation were positive in 3 cases (3.0\%), ST depression, ventricular fibrillation, 2nd degree heart block and polymorphic ventricular tachycardia were positive in one case only $(1.0 \%)$. While in control group, QTc prolongation was positive in 2 cases $(4.0 \%)$, ST elevation, inverted T wave, ST depression and ventricular fibrillation were positive in one case only.

There was a statistically significant difference in ECG abnormalities between the studied groups $(\mathrm{P}<0.05)$ (Table 2 b).

Comparisons between biochemical investigations findings in the studied groups:

There was no significant difference between cases and controls groups as regards, fasting blood sugar, complete blood count ( $\mathrm{Hb}, \mathrm{RPCs}, \mathrm{WBCs}$ and Hematocrit value), blood urea and serum potassium $(\mathrm{P}>0.05)$.
A significant difference was observed (detected) between cases and controls groups $(\mathrm{P}<0.05)$ : in the study group the mean serum creatinine was $(1.76 \pm 0.31)$, serum uric acid was $(4.11 \pm 1.17)$, arterial blood gases $(\mathrm{PH}$, $\mathrm{HCO} 3, \mathrm{PaO} 2$ and $\mathrm{PaCO} 2)$ were $(7.15 \pm 0.17,21.11 \pm 2.16,70.11 \pm 8.17$ and $47.4 \pm 2.77$ respectively), serum electrolytes (sodium, chloride, and bicarbonate $) \quad$ were $\quad(130.11 \pm 6.49$, $106.5 \pm 4.1$ and $21.4 \pm 2.01$ respectively), reduction in normal cholinesterase level (mild, moderate and severe) were $(390.11 \pm 229.91,250.5 \pm 100.99$ and $169 \pm 20.11$ respectively), cardiac enzyme (Troponin, CK-MB, LDH, $\mathrm{AST}$, and $\mathrm{Mb})$ were $(91.0 \pm 0.12$, $80.12 \pm 11.51,70.12 \pm 11.51,50.12 \pm 12.11$ and $\quad 86.12 \pm 11.3$ respectively), Phosphoro di thionate and Diethyl paranitrophenyl phosphate were $(53.6 \pm 80.7$ and 30.16 \pm 49.61 respectively). While in controls groups: the mean serum creatinine was $(1.03 \pm 0.10)$, serum uric acid was $(5.01 \pm$ $1.82)$, arterial blood gases ( $\mathrm{PH}, \mathrm{HCO} 3$, $\mathrm{PaO} 2$ and $\mathrm{PaCO} 2)$ were $(7.35 \pm 0.10$, $24.01 \pm 1.72, \quad 95.01 \pm 4.82$ and 40.01 4 4.02 respectively), serum electrolytes (sodium, chloride, and bicarbonate) were $(135.95 \pm$ 5.91, $101.5 \pm 5.1$ and $20.4 \pm 2.01$ respectively), cholinesterase level was $(3500 \pm 100.12)$, cardiac enzyme (CK-MB, LDH, AST, and $\mathrm{Mb})$ were $(50.33 \pm 30.63,45.33 \pm$ 10.83, 30.12 \pm 11 . 2, 30. $21 \pm 10.4$ and 30 . $21 \pm 10.4$ respectively) and Phosphor dithionate and Diethyl para-nitrophenyl

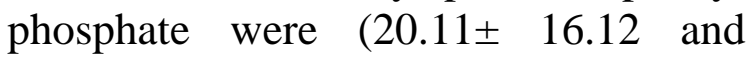
$10.44 \pm 9.22$ respectively). Significant difference was detected between observed between cases and controls groups $(\mathrm{P}<0.05)$ in blood gases, sodium, chloride, bicarbonate, cholinesterase and cardiac biomarker. (Table 3). 
Percentage of pesticide exposure and cholinesterase level in the study cases:

As regarding percent of pesticide exposure finding in the study group; phosphor dithionate (Malathion) is positive in 70 cases $(70.0 \%)$ and Diethyl para-nitrophenyl phosphate (DPT) is positive in 62 cases $(62.0 \%)$, while in control group phosphor dithionate (Malathion) is positive in 2 cases $(4.0 \%)$ and Diethyl paranitrophenyl phosphate (DPT) is positive in 3 cases $(6.0 \%)$. There was an extremely significant difference between studied groups $(\mathrm{P}<0.005)$

As regarding percent of the reduction in cholinesterase level in the study group; mild decrease in 50 cases $(50.0 \%)$, a moderate decrease in 40 cases $(40.0 \%)$ and severe decrease in 10 cases $(10.0 \%)$, while in control group mild decrease in one case $(2.0 \%)$. There was an extremely significant difference between studied groups $(\mathrm{P}<0.005)$. (Table 4$)$.

Correlation between pesticide exposures and cardiac and biochemical abnormalities.

-Negative correlation in the studied groups of pesticide exposures with fasting blood sugar, CBC (Hb, RPCs and WBCs) blood urea level, serum chloride, and ECG changes (T inversion, ST depression, atrial extrasystole, ventricular fibrillation, 2nd degree heart block and polymorphic ventricular tachycardia) and its non-significant $(\mathrm{P}>0.05)$.

-The positive correlation in the study group of pesticide exposures with hematocrit value, serum uric acid, serum electrolytes (sodium and bicarbonate) and 1st-degree heart block and it's also non-significant $(\mathrm{P}>0.05)$.

-Negative correlation in the control group of pesticide exposures with CBC, blood urea, serum creatinine, serum uric acid, arterial blood gases, serum electrolytes, reduction in cholinesterase level, ECG changes and it's also nonsignificant $(\mathrm{P}>0.05)$.

-The positive correlation in the study group of pesticide exposures with serum creatinine, arterial blood gases, serum potassium, moderate and severe reduction in cholinesterase level, cardiac enzyme, (QTc prolongation, ST elevation, and ventricular extrasystole) and its significant $(\mathrm{P}<0.05)$. (Table 5). 
Table (1): Comparison between study and control group as regard demographic and social data byChi-square $\left(\chi^{2}\right)$ test.

\begin{tabular}{|c|c|c|c|c|}
\hline \multicolumn{2}{|c|}{ Studied Parameters } & $\begin{array}{c}\text { Study group } \\
(\mathrm{n}=100)\end{array}$ & $\begin{array}{c}\text { Control group } \\
(n=50)\end{array}$ & P value \\
\hline \multirow{2}{*}{ Sex n. $(\%)$} & Male & $70(70.0 \%)$ & $33(66.0 \%)$ & \multirow{2}{*}{$\begin{array}{l}0.789 \\
(\mathrm{NS})\end{array}$} \\
\hline & Female & $30(30 \%)$ & $17(34 \%)$ & \\
\hline \multicolumn{2}{|c|}{ Age $($ mean \pm SD) in years $n .(\%)$} & $(25.10 \pm 01 \%)$ & $(20.05 \pm 01 \%)$ & $\begin{array}{l}0.410 \\
\text { (NS) }\end{array}$ \\
\hline \multirow{5}{*}{ Occupation } & Farmers & $66(66.0 \%)$ & $10(20.0 \%)$ & \multirow{5}{*}{$\begin{array}{l}0.620 \\
(\mathrm{NS})\end{array}$} \\
\hline & Skilled laborer & $16(16.0 \%)$ & $30(60.0 \%)$ & \\
\hline & Student & $10(10.0 \%)$ & $6(12.0 \%)$ & \\
\hline & Civil employed & $4(4.0 \%)$ & $1(2.0 \%)$ & \\
\hline & Not employed & $4(4.0 \%)$ & $3(6.0 \%)$ & \\
\hline \multirow{2}{*}{$\begin{array}{c}\text { Region of } \\
\text { current } \\
\text { residence n. }(\%)\end{array}$} & Urban & $10(10.0 \%)$ & $30(60.0 \%)$ & \multirow{2}{*}{$0.004(\mathrm{~S})$} \\
\hline & Rural & $90(90.0 \%)$ & $20(40 \%)$ & \\
\hline \multirow{3}{*}{$\begin{array}{l}\text { Intention of } \\
\text { poisoning }\end{array}$} & Suicidal & $28(28.0 \%)$ & $1(2.0 \%)$ & \multirow{3}{*}{$0.003(\mathrm{~S})$} \\
\hline & Homicidal & $7(7.0 \%)$ & $3(6.0 \%)$ & \\
\hline & Accidental & $65(65.0 \%)$ & $3(6.0 \%)$ & \\
\hline \multirow{3}{*}{ Cases severity } & Mild & $30(30.0 \%)$ & $2(4.0 \%)$ & \multirow{2}{*}{$0.001(\mathrm{~S})$} \\
\hline & Moderate & $60(60.0 \%)$ & $2(4.0 \%)$ & \\
\hline & Severe & $10(10.0 \%)$ & $3(6.0 \%)$ & \\
\hline \multirow{3}{*}{$\begin{array}{l}\text { Time since last } \\
\text { intake and } \\
\text { admission } \\
\text { (days) }\end{array}$} & 24 hours $<$ & $65(65.0 \%)$ & $3(6.0 \%)$ & \multirow{3}{*}{$0.002(\mathrm{~S})$} \\
\hline & 1-3 days & $32(32.0 \%)$ & $4(8.0 \%)$ & \\
\hline & $>3$ days & $3(3.0 \%)$ & $0(0.0 \%)$ & \\
\hline \multirow{4}{*}{$\begin{array}{l}\text { Smoker index } \mathrm{n} . \\
(\%)\end{array}$} & Non & $15(15.0 \%)$ & $5(10.0 \%)$ & \multirow{4}{*}{$0.001(\mathrm{~S})$} \\
\hline & Mild & $25(25.0 \%)$ & $7(14.0 \%)$ & \\
\hline & Moderate & $24(24.0 \%)$ & $24(48.0 \%)$ & \\
\hline & Severe & $36(36.0 \%)$ & $14(28.0 \%)$ & \\
\hline \multirow{2}{*}{$\begin{array}{c}\text { Past history n. } \\
(\%)\end{array}$} & Bilharziasis & $25(25.0 \%)$ & $2(4.0 \%)$ & \multirow{2}{*}{$<0.05(\mathrm{~S})$} \\
\hline & Diabetes & $10(10.0 \%)$ & $3(6.0 \%)$ & \\
\hline \multirow{3}{*}{$\begin{array}{c}\text { Agricultural } \\
\text { activities n. }(\%)\end{array}$} & $\begin{array}{l}\text { Working or } \\
\text { helping }\end{array}$ & $25(25.0 \%)$ & $3(6.0 \%)$ & \multirow{3}{*}{$0.001(\mathrm{~S})$} \\
\hline & Exposures & $30(30.0 \%)$ & $1(2.0 \%)$ & \\
\hline & Crops grown & $30(30.0 \%)$ & $1(2.0 \%)$ & \\
\hline \multicolumn{2}{|c|}{ Wearing protective clothes $\mathrm{n} .(\%)$} & $15(15.0 \%)$ & $2(4.0 \%)$ & $\begin{array}{c}0.310 \\
(\mathrm{NS})\end{array}$ \\
\hline
\end{tabular}

$S=$ Significant. NS = Non -significant. Significant at $P<0.05$. Non-significant difference at $\mathbf{P}>\mathbf{0 . 0 5}$ 
Table (2a): Clinical manifestations of the studied groups by students ( $\mathrm{t}$ ) test.

\begin{tabular}{|c|c|c|c|c|}
\hline \multicolumn{2}{|r|}{ Studied Parameters } & $\begin{array}{c}\text { Study group } \\
(n=100)\end{array}$ & $\begin{array}{l}\text { Control group } \\
(n=50)\end{array}$ & $P$ value \\
\hline \multirow{5}{*}{$\begin{array}{l}\text { Vital } \\
\text { sign }\end{array}$} & $\begin{array}{l}\text { Systolic blood pressure } \\
(\text { mean } \pm \text { SD })\end{array}$ & $100.80 \pm 10.77$ & $120.56 \pm 6.53$ & $0.087(\mathrm{NS})$ \\
\hline & $\begin{array}{c}\text { Diastolic blood pressure } \\
(\text { mean } \pm \text { SD })\end{array}$ & $60.77 \pm 10.16$ & $80.25 \pm 6.05$ & $0.118(\mathrm{NS})$ \\
\hline & Temperature ${ }^{\circ} \mathrm{C}$ & $36.12 \pm 1.06$ & $37.53 \pm 1.61$ & $0.111(\mathrm{NS})$ \\
\hline & Respiratory rate/min & $20.32 \pm 1.06$ & $18.39 \pm 1.61$ & $0.013(\mathrm{~S})$ \\
\hline & Heart rate beats/min & $50.97 \pm 5.70$ & $62.33 \pm 6.04$ & $0.012(\mathrm{~S})$ \\
\hline
\end{tabular}

$\mathbf{S}=$ Significant. NS = Non significant. Significant at $\mathbf{P}<\mathbf{0 . 0 5}$. Non-significant difference at $\mathbf{P}>\mathbf{0 . 0 5}$.

Table (2b): Clinical finding of chest X-ray and ECG changes in the studied groups by students $(\mathrm{t})$ test.

\begin{tabular}{|c|c|c|c|c|}
\hline \multicolumn{2}{|c|}{ Studied Parameters } & $\begin{array}{l}\text { Study group } \\
\quad(n=100)\end{array}$ & $\begin{array}{l}\text { Control group } \\
\qquad(\mathbf{n}=\mathbf{5 0})\end{array}$ & P value \\
\hline \multicolumn{2}{|c|}{$\begin{array}{c}\text { Positive Chest X-ray (pulmonary } \\
\text { edema) }\end{array}$} & $50(50.0 \%)$ & $0(0.0 \%)$ & $0.002(\mathrm{~S})$ \\
\hline \multirow{10}{*}{$\begin{array}{c}\text { ECG } \\
\text { changes }\end{array}$} & QTe prolongation & $50(50.0 \%)$ & $2(4.0 \%)$ & \multirow{10}{*}{$\begin{array}{l}\mathrm{P}<0.05 \\
\quad(\mathrm{~S})\end{array}$} \\
\hline & ST elevation & $3(3.0 \%)$ & $1(2.0 \%)$ & \\
\hline & $\mathrm{T}$ inversion & $5(5.0 \%)$ & $1(2.0 \%)$ & \\
\hline & ST depression & $1(1.0 \%)$ & $1(2.0 \%)$ & \\
\hline & Atrial extrasystole & $4(4.0 \%)$ & $0(0.0 \%)$ & \\
\hline & Ventricular fibrillation & $1(1.0 \%)$ & $1(2.0 \%)$ & \\
\hline & Ventricular extrasystole & $20(20.0 \%)$ & $0(0.0 \%)$ & \\
\hline & 1st degree heart block & $8(8.0 \%)$ & $0(0.0 \%)$ & \\
\hline & 2nd degree heart block & $1(1.0 \%)$ & $0(0.0 \%)$ & \\
\hline & $\begin{array}{l}\text { Polymorphic ventricular } \\
\text { tachy }\end{array}$ & $1(1.0 \%)$ & $0(0.0 \%)$ & \\
\hline
\end{tabular}

$\mathrm{S}=$ Significant. NS = Non significant. Significant at $\mathrm{P}<0.05$. Non-significant difference at $\mathbf{P}>\mathbf{0 . 0 5}$.

Table (3): Comparisons between chemical investigations finding in the studied groups

by students $(\mathrm{t})$ test.

\begin{tabular}{|c|c|c|c|c|c|c|}
\hline \multicolumn{2}{|c|}{ Tests Groups } & \multicolumn{2}{|c|}{$\begin{array}{c}\text { Study group } \\
(n=100)\end{array}$} & \multicolumn{2}{|c|}{$\begin{array}{c}\text { Control group } \\
(\mathbf{n}=\mathbf{2 0})\end{array}$} & \multirow[t]{2}{*}{ P value } \\
\hline & & $\mathbf{M}$ & $\pm \mathrm{SD}$ & $\mathbf{M}$ & $\pm \mathrm{SD}$ & \\
\hline \multicolumn{2}{|c|}{ Fasting blood sugar (mg/dL) } & 85.22 & 8.21 & 60.77 & 5.46 & $0.801(\mathrm{NS})$ \\
\hline \multirow{4}{*}{$\mathbf{C B C}$} & $\mathrm{Hb}$ gm\% & 12.5 & 1.82 & 13.5 & 1.02 & \multirow{4}{*}{$\begin{array}{l}\mathrm{P}>0.05(\mathrm{NS} \\
)\end{array}$} \\
\hline & RBCs x 106/ml & 3.97 & 1.91 & 4.78 & 1.01 & \\
\hline & WBCs x $103 / \mathrm{ml}$ & 4.27 & 2.91 & 6.77 & 3.01 & \\
\hline & Hematocrit value vol\% & 32.9 & 1.11 & 40.10 & 5.66 & \\
\hline \multicolumn{2}{|c|}{ Blood urea (mg/dL) } & 33.31 & 6.37 & 32.54 & 7.16 & $0.504(\mathrm{NS})$ \\
\hline \multicolumn{2}{|c|}{ Serum creatinine $(\mathrm{mg} / \mathrm{dL})$} & 1.76 & 0.31 & 1.03 & 0.10 & $0.022(\mathrm{~S})$ \\
\hline \multicolumn{2}{|c|}{ Serum uric acid (mg/dL) } & 4.11 & 1.17 & 5.01 & 1.82 & $0.030(\mathrm{~S})$ \\
\hline
\end{tabular}




\begin{tabular}{|c|c|c|c|c|c|c|}
\hline \multirow{4}{*}{ ABG } & Blood PH & 7.15 & 0.17 & 7.35 & 0.10 & \multirow{4}{*}{$\begin{array}{l}\mathrm{P}<0.05 \\
(\mathrm{~S})\end{array}$} \\
\hline & $\mathrm{HCO} 3 \mathrm{meq} / \mathrm{L}$ & 21.11 & 2.16 & 24.01 & 1.72 & \\
\hline & $\mathrm{PaO} 2 \mathrm{mmHg}$ & 70.11 & 8.17 & 95.01 & 4.82 & \\
\hline & $\mathrm{PaCO} 2 \mathrm{mmHg}$ & 47.4 & 2.77 & 40.01 & 4.02 & \\
\hline \multirow{4}{*}{$\begin{array}{l}\text { Serum } \\
\text { electrolytes }\end{array}$} & Sodium $(\mathrm{mmol} / \mathrm{L})$ & 130.2 & 6.49 & 135.95 & 5.91 & $\begin{array}{l}\mathrm{P}< \\
0.001(\mathrm{~S})\end{array}$ \\
\hline & Potassium $(\mathrm{mmol} / \mathrm{L})$ & 4.5 .1 & 2.01 & 3.5 & 2.2 & $\begin{array}{l}\mathrm{P}>0.05 \\
(\mathrm{NS})\end{array}$ \\
\hline & Chloride (mmol/L). & 106.5 & 4.1 & 101.5 & 5.1 & $\begin{array}{l}\mathrm{P}< \\
0.001(\mathrm{~S})\end{array}$ \\
\hline & Bicarbonate $(\mathrm{mmol} / \mathrm{L})$ & 21.4 & 2.01 & 20.4 & 2.01 & $\begin{array}{l}\mathrm{P}< \\
0.001(\mathrm{~S})\end{array}$ \\
\hline \multirow{3}{*}{$\begin{array}{l}\text { Decrease in } \\
\text { cholinesterase } \\
\text { level }\end{array}$} & Mild =380-760 IU/L & 390 & 229.91 & 3500 & 100.12 & \multirow{3}{*}{$0.043(\mathrm{~S})$} \\
\hline & $\begin{array}{l}\text { Moderate }=190-380 \\
\text { IU/L }\end{array}$ & 250 & 100.99 & 3500 & 100.12 & \\
\hline & Severe $=<190 \mathrm{IU} / \mathrm{L}$ & 169 & 20.11 & 3500 & 100.12 & \\
\hline \multirow{5}{*}{$\begin{array}{l}\text { Cardiac } \\
\text { enzyme }\end{array}$} & Troponin $\mu \mathrm{g} / \mathrm{mL}$ & 91.0 & 0.12 & $\begin{array}{c}0 \\
(0.0 \%)\end{array}$ & 0.0 & \multirow{5}{*}{$\mathrm{P}<0.05(\mathrm{~S})$} \\
\hline & CK-MB U/L & 80.1 & 10.51 & 50.33 & 30.63 & \\
\hline & LDH U/L & 70.12 & 11.51 & 45.33 & 10.83 & \\
\hline & AST U/L & 50.12 & 12.11 & 30.12 & 11.2 & \\
\hline & $\mathrm{Mb} \mu \mathrm{g} / \mathrm{ml}$ & 86.12 & 11.3 & 30.21 & 10.4 & \\
\hline \multicolumn{2}{|c|}{ Phosphoro di thionate (PDT) $\mu \mathrm{g} / \mathrm{L}$} & 53.6 & 80.7 & 20.11 & 16.12 & \multirow[b]{2}{*}{$\mathrm{P}<0.05(\mathrm{~S})$} \\
\hline \multicolumn{2}{|c|}{$\begin{array}{l}\text { Diethyl paranitrophenyl phosphate } \\
\text { (DPT) } \mu \mathrm{g} / \mathrm{L}\end{array}$} & 30.16 & 49.61 & 10.44 & 9.22 & \\
\hline
\end{tabular}

$\mathrm{S}=$ Significant. NS $=$ Non significant. Significant at $\mathrm{P}<0.05$. Non-significant difference at $\mathbf{P}>\mathbf{0 . 0 5}$

Table (4): Percentage of pesticide exposure and cholinesterase level in the study cases by Chi square $(\chi 2)$ tests.

\begin{tabular}{|c|c|c|c|c|}
\hline \multicolumn{2}{|l|}{ Tests } & $\begin{array}{c}\text { Study } \\
\text { group } \\
(\mathbf{n}=100)\end{array}$ & $\begin{array}{c}\text { Control } \\
\text { group }(n=50)\end{array}$ & P value \\
\hline \multicolumn{2}{|c|}{ Phosphor dithionate (Malathion) n. (\%) } & $70(70.0 \%)$ & $2(4.0 \%)$ & $\begin{array}{l}0.003 \\
(\mathrm{~S})\end{array}$ \\
\hline \multicolumn{2}{|c|}{$\begin{array}{c}\text { Diethyl para-nitrophenyl phosphate (DPT) } \\
\text { n. }(\%)\end{array}$} & $62(62.0 \%)$ & $3(6.0 \%)$ & $\begin{array}{l}0.001 \\
(\mathrm{~S})\end{array}$ \\
\hline \multirow{3}{*}{$\begin{array}{c}\text { Decrease in } \\
\text { cholinesterase } \\
\text { level }\end{array}$} & Mild =380-760 IU/L & $50(50.0 \%)$ & $1(2.0 \%)$ & \multirow{3}{*}{$\begin{array}{l}0.011 \\
(\mathrm{~S})\end{array}$} \\
\hline & Moderate $=190-380 \mathrm{IU} / \mathrm{L}$ & $40(40.0 \%)$ & $0(0.0 \%)$ & \\
\hline & Severe $=<190 \mathrm{IU} / \mathrm{L}$ & $10(10.0 \%)$ & $0(0.0 \%)$ & \\
\hline
\end{tabular}

$\mathrm{S}=$ Significant. Significant at $\mathrm{P}<\mathbf{0 . 0 5}$. Non-significant difference at $\mathbf{P}>0.05$ 
Table (5): Correlation between pesticide exposures and cardiac and some biochemical abnormalities.

\begin{tabular}{|c|c|c|c|c|c|}
\hline \multicolumn{2}{|c|}{$\begin{array}{ll}\text { Tests } & \text { Groups } \\
\end{array}$} & \multicolumn{2}{|c|}{$\begin{array}{c}\text { Study group } \\
(\mathrm{n}=100)\end{array}$} & \multicolumn{2}{|c|}{$\begin{array}{c}\text { Control group } \\
(\mathbf{n}=\mathbf{5 0})\end{array}$} \\
\hline & & $\mathrm{r}$ & $\mathrm{P}$ & $\mathrm{r}$ & $\mathrm{P}$ \\
\hline \multicolumn{2}{|c|}{ Fasting blood sugar (mg/dL) } & -0.33 & $0.07(\mathrm{NS})$ & -0.1 & $0.47(\mathrm{NS})$ \\
\hline \multirow[t]{4}{*}{$\mathrm{CBC}$} & $\mathrm{Hb}$ gm $\%$ & -0.22 & $0.05(\mathrm{NS})$ & $0.61-$ & $0.61(\mathrm{NS})$ \\
\hline & RBCs x $106 / \mathrm{ml}$ & $0.35-$ & $0.03(\mathrm{~S})$ & -0.86 & $0.66(\mathrm{NS})$ \\
\hline & WBCs $\times 103 / \mathrm{ml}$ & $0.66-$ & $0.88(\mathrm{NS})$ & $0.62-$ & $0.62(\mathrm{NS})$ \\
\hline & Hematocrit value vol\% & 0.04 & $0.06(\mathrm{NS})$ & $0.71-$ & $0.72(\mathrm{NS})$ \\
\hline \multicolumn{2}{|c|}{ Blood urea $(\mathrm{mg} / \mathrm{dL})$} & -3.31 & $0.71(\mathrm{NS})$ & -0.6 & $0.41(\mathrm{NS})$ \\
\hline \multicolumn{2}{|c|}{ Serum creatinine $(\mathrm{mg} / \mathrm{dL})$} & 0.26 & $0.01(\mathrm{~S})$ & -0.03 & $0.13(\mathrm{NS})$ \\
\hline \multicolumn{2}{|c|}{ Serum uric acid $(\mathrm{mg} / \mathrm{dL})$} & 4.11 & $0.55(\mathrm{NS})$ & -0.66 & $0.86(\mathrm{NS})$ \\
\hline \multirow{4}{*}{$\mathrm{ABG}$} & Blood PH & 0.02 & $0.02(\mathrm{~S})$ & -0.13 & $0.73(\mathrm{NS})$ \\
\hline & $\mathrm{HCO} 3 \mathrm{meq} / \mathrm{L}$ & 0.07 & $0.01(\mathrm{~S})$ & $0.47-$ & $0.47(\mathrm{NS})$ \\
\hline & $\mathrm{PaO} 2 \mathrm{mmHg}$ & 0.22 & $0.04(\mathrm{~S})$ & $0.61-$ & $0.61(\mathrm{NS})$ \\
\hline & $\mathrm{PaCO} 2 \mathrm{mmHg}$ & 0.35 & $0.03(\mathrm{~S})$ & $0.86-$ & $0.86(\mathrm{NS})$ \\
\hline \multirow{4}{*}{$\begin{array}{l}\text { Serum } \\
\text { electrolyt } \\
\text { es }\end{array}$} & Sodium $(\mathrm{mmol} / \mathrm{L})$ & 0.26 & $0.08(\mathrm{NS})$ & -0.62 & $0.62(\mathrm{NS})$ \\
\hline & Potassium (mmol/L) & 0.01 & $0.01(\mathrm{~S})$ & -0.53 & $0.21(\mathrm{NS})$ \\
\hline & Chloride (mmol/L). & -0.23 & $0.41(\mathrm{NS})$ & -0.52 & $0.15(\mathrm{NS})$ \\
\hline & Bicarbonate $(\mathrm{mmol} / \mathrm{L})$ & 21.4 & $0.11(\mathrm{NS})$ & -0.14 & $0.31(\mathrm{NS})$ \\
\hline \multirow{3}{*}{$\begin{array}{l}\text { Decrease } \\
\text { in } \\
\text { cholineste } \\
\text { rase level }\end{array}$} & Mild =380-760 IU/L & -0.01 & $0.01(\mathrm{~S})$ & -0.12 & $0.12(\mathrm{NS})$ \\
\hline & Moderate $=190-380 \mathrm{IU} / \mathrm{L}$ & 0.02 & $0.02(\mathrm{~S})$ & -0.24 & $0.10(\mathrm{NS})$ \\
\hline & Severe $=<190 \mathrm{IU} / \mathrm{L}$ & 0.03 & $0.03(\mathrm{~S})$ & -0.25 & $0.60(\mathrm{NS})$ \\
\hline \multirow{5}{*}{$\begin{array}{l}\text { Cardiac } \\
\text { enzyme }\end{array}$} & Troponin $\mu \mathrm{g} / \mathrm{mL}$ & 0.01 & $0.02(\mathrm{~S})$ & $0.01-$ & $0.90(\mathrm{NS})$ \\
\hline & CK-MB U/L & 0.02 & $0.05(\mathrm{NS})$ & $0.01-$ & $0.63(\mathrm{NS})$ \\
\hline & LDH U/L & 0.04 & $0.01(\mathrm{~S})$ & $0.01-$ & $0.83(\mathrm{NS})$ \\
\hline & AST U/L & 0.13 & $0.03(\mathrm{~S})$ & $0.01-$ & $0.72(\mathrm{NS})$ \\
\hline & $\mathrm{Mb} \mu \mathrm{g} / \mathrm{ml}$ & 0.02 & $0.04(\mathrm{~S})$ & $0.01-$ & $0.41(\mathrm{NS})$ \\
\hline \multirow{10}{*}{$\begin{array}{r}\text { ECG } \\
\text { changes }\end{array}$} & QTc prolongation & 0.02 & $0.01(\mathrm{~S})$ & -0.21 & $0.13(\mathrm{NS})$ \\
\hline & ST elevation & 0.12 & $0.01(\mathrm{~S})$ & -0.15 & $0.72(\mathrm{NS})$ \\
\hline & $\mathrm{T}$ inversion & $0.20-$ & $0.11(\mathrm{NS})$ & -0.23 & $0.83(\mathrm{NS})$ \\
\hline & ST depression & $0.32-$ & $0.21(\mathrm{NS})$ & -0.52 & $0.77(\mathrm{NS})$ \\
\hline & Atrial extrasystole & $0.41-$ & $0.41(\mathrm{NS})$ & -0.11 & $0.83(\mathrm{NS})$ \\
\hline & Ventricular fibrillation & $0.12-$ & $0.81(\mathrm{NS})$ & -0.35 & $0.78(\mathrm{NS})$ \\
\hline & Ventricular extrasystole & 0.02 & $0.04(\mathrm{~S})$ & -0.25 & $0.33(\mathrm{NS})$ \\
\hline & $1^{\text {st }}$ degree heart block & 0.02 & $0.91(\mathrm{NS})$ & -0.24 & $0.22(\mathrm{NS})$ \\
\hline & $2^{\text {nd }}$ degree heart block & $0.12-$ & $0.88(\mathrm{NS})$ & -0.22 & $0.53(\mathrm{NS})$ \\
\hline & $\begin{array}{l}\text { Polymorphic ventricular } \\
\text { tachy }\end{array}$ & $0.04-$ & $0.98(\mathrm{NS})$ & -0.13 & $0.72(\mathrm{NS})$ \\
\hline
\end{tabular}

r: positive correlation. r-; negative correlation. Significant at $P<0.05$. Non-significant difference at $\mathbf{P}>0.05$. 


\section{DISCUSSION}

Pesticides are chemical or biological substances used to kill or repel targeted organisms. All pesticides are poisons. In many cases, they are designed to impact the immune, reproductive, cardiac or nervous system of insects (Abby and Sarah, 2012).

Pesticides are mainly utilized in agriculture to increase agricultural yield for crop protection. Pesticides are also applied in homes and gardens. More than 140.000 tonnes of pesticides are used annually in the European Union for agricultural purposes only (Ramazzini, 2012).

OP compounds poisoning is always considered a life-threatening condition. The mechanism by which organophosphates induce cardiotoxicity is still uncertain (Taira et al., 2006); (Yurumez et al., 2006).

Cardiac toxicity after OP compounds poisoning may occur in three phases. Initially, there is a brief period of increased sympathetic activity characterized by tachycardia and hypertension. This is followed by a more prolonged period of extreme cholinergic activity during which bradycardia and hypotension along with ST-T changes and life-threatening rhythm disturbances can occur. A third longer phase is usually associated with a prolonged QT interval and polymorphic ventricular tachycardia that can result in sudden death (Ludomirsky et al., 2006).

So the objective of this work was to study the possible correlation between acute exposure to some (OP) as phosphor dithionate (PDT=Malathion) and diethyl para-nitrophenyl phosphate parathion (DPT=Falidol or Police ElNagda) and occurrence of cardiac and some biochemical markers abnormalities
The study was conducted from the $1^{\text {st }}$ of December 2016 to $1^{\text {st }}$ of October 2017. It involved 100 cases who recruited from the emergency department of Al-Azhar University hospital (New Damietta) and emergency hospital at Mansoura University. In addition to 50 healthy subjects as a control group.

As regards the demographic data of the studied cases, $(70.0 \%)$ were males and $(30 \%)$ were females in the study group.

The majority of male cases is due to the fact that male is more likely to report the use of pesticide in agriculture which is accepted in the society (Mucci et al., 2001).

Male dominance in pesticides poisoning was also recorded by (Milena et al., 2015).

As regards the mean age of poisoning it was $(25.10 \pm 01 \%)$ in the study group. These findings signify an alarming trend in the prevalence of pesticides exposure in such age. In a prospective study over a course of twelve months by Rafighdoost et al., (2015), of 51 patients (21males) with OP poisoning, 19 patients were in the 15 to 24 age group, and followed by 17 patients were in the 25 to 45 age group.

As regards job engagement in the study group, farmers were $(66.0 \%)$, the skilled laborer were $(16.0 \%)$, the students were $(10.0 \%)$, civil and not employed cases were $(4.0 \%)$ each.

Different explanations were proposed for each category e.g. farmers and skilled laborer may be exposing more to OP toxicity due to a prolonged time off work. Also, nonemployer may expose OP toxicity as a process of suicidal attempts.

As regards residence distribution in the study group, urban was (10.0\%) and rural were $(90.0 \%)$. These results were 
in agreement with Lacasana et al., (2015) as reported that the farming workers are the group with the maximum exposure to the pesticide.

As regards manner of poisoning in the study group, suicidal were $(28.0 \%)$, homicidal were $(7.0 \%)$ and accidental were $(65.0 \%)$. \%). Also Rafighdoost et al., (2015), reported that $78.43 \%$ of patients had suicidal exposure. Also, Jihlin et al's study on 4799 cases of OP exposure, 3106 cases $(64.72 \%)$ was suicidal, and only 759 cases (15.82\%) were occupational (Lin et al., 2015).

The severity of cases in the study group was mild $(30.0 \%)$, moderate were $(60.0 \%)$ and severe were $(10.0 \%)$. These results are in accordance with Laudari et al., (2014), as it is reported that, the severity of poisoning was moderate in (67.8\%).

As regards the time elapsed since last intake and admission in the study group; less than 24 hours were $(65.0 \%)$, from 1 to three days were $(32.0 \%)$ and more than 3 days in 3 cases $(3.0 \%)$. Also Laudari et al., (2014), was reported that elapsed time between poisoning and admission, as time less than 2 hours were (22.6\%), 2-6 hours were $(66.1 \%)$ and more than 6 hours were $(11.3 \%)$.

As regards smoker index in the study group; the number of non-smokers was $(15.0 \%)$, mild smokers was $(25.0 \%)$, moderate was $(24.0 \%)$ and heavy was $(36.0 \%)$, while smoker index in the control group, number of nonsmokers was $(10.0 \%)$, mild smokers was $(14.0 \%)$, moderate was $(48.0 \%)$ and heavy was $(28.0 \%)$. This observation was recorded Andersson, (2009).

In the study group, past history bilharziasis was $(25.0 \%)$ and diabetes was $(10.0 \%)$. The result of diabetes was in agreement with $\mathbf{O m}$ and Ganda, (2016), as reported that diabetic patients have a higher prevalence of cardiac disorders compared with the normal population.

Agricultural activities in the study group, working or helping were $(25.0 \%)$, exposures and crops grown were $(30.0 \%)$ each.

A number of cases wearing protective clothing in the study group were $(15.0 \%)$ and was $(4.0 \%)$ in the control group. These are in accordance with (Xu et al., (2013).

As regarding the clinical data, there is no significant difference between cases and controls as regard mean systolic and diastolic blood pressure and temperature as $(\mathrm{P}>0.05)$.

While there was significant difference between studied groups as regard (respiratory and heart rates, pulmonary edema by $\mathrm{X}$ ray and ECG changes (QTc prolongation was positive in $(50.0 \%)$, ventricular extrasystole were positive in $(20.0 \%), 1^{\text {st }}$ degree heart block were positive in $(8.0 \%)$, inverted $\mathrm{T}$ wave were positive in $(5.0 \%)$, atrial extrasystole were positive in $(4.0 \%)$, ST elevation were positive in $(3.0 \%)$, ST depression, ventricular fibrillation, $2^{\text {nd }}$ degree heart block and polymorphic ventricular tachycardia were positive in one case only (1.0\%). Also Karki et al., (2014) and Yurumez et al., (2015), found that respiratory failure and ventricular fibrillation in 3 patients (2.6\%) each, aspiration pneumonia and status epilepticus in $1(0.9 \%)$ each.

The cardiac effect was also the most common finding in studies done in Nepal, Turkey and India Paul and Bhattacharyya, (2012), though, its frequency was higher $(37.8 \%, 55.5 \%$, $62.5 \%$, respectively). In overall, the frequency of QTc prolongation in several series of severe OP poisoning was shown to be $20-80 \%$ depending on the severity of the intoxication and the 
type of the toxic agent Kiss, and Fazekas, (2011) and Lyzhnikov et al., (2014),

Karki et al. reported that sinus tachycardia observed in $40.5 \%$ of patients Eddleston, (2010). Yurumez et al. reported that sinus tachycardia was a more common finding (in $31.8 \%$ cases) in their study (Ludomirsky et al., 2014). In another study, Saadeh et al. reported that sinus tachycardia was reported in $35.0 \%$ of cases (Senanayake et al., 2014). Although non-specific ST-T changes have generally been recognized as not being directly related to any cardiac diseases, they also have been seen before ST elevation associated with coronary spasm (Megarbane, 2014). Thus, the nonspecific ST-T change for the patient with (OP) poisoning has a considerable clinical sense (Senanayake et al., 2014). Beside, a large QT dispersion is a result of the ischemic change which may conceal the QT prolongation in the affecting vascular area (10). Also, Rafigh Doost et al's study in Iran, $64.71 \%$ of the patients had a prolonged QTc interval Yurumez et al., (2015). Karki et al. also seen a prolonged QTc interval in 14 patients $(37.8 \%)$, ST-T changes in 11 cases $(29.7 \%)$, and conduction defects in two cases $(5.4 \%)$. Chuang et al. determined that 97 (43.5\%) patients had QTc prolongation, and these patients had a poor prognosis. Jang et al. estimated that 67 of 170 patients had QTc prolongation and in this group, mortality rate, respiratory failure rate, and frequency of ventricular premature contractions were significantly higher than those of patients without QTc prolongation (Saadeh et al., 2014). So in this study as compared with the other studies, QTc prolongation occurred in 50 patients (50.0\%) with OP poisoning.
The present study showed that there was no significant difference between cases and controls groups as regard; fasting blood sugar, CBC ( $\mathrm{Hb}$, RPCs, WBCs and Hematocrit value), blood potassium level $(\mathrm{P}>0.05)$.

While there was a significant difference between cases and controls groups as regard; serum creatinine, serum uric acid, $\mathrm{ABG}(\mathrm{PH}, \mathrm{HCO} 3$, $\mathrm{PaO} 2$ and $\mathrm{PaCO} 2$ ), serum electrolytes (sodium, chloride and bicarbonate), decreasing in level of cholinesterase enzyme, cardiac enzyme (Troponin, CK-MB, LDH, AST and Mb) and OP compounds (phosphor dithionate and diethyl para-nitrophenyl phosphate) as $(\mathrm{P}<0.05)$. These results are in agreements with Karki et al., (2014) and Bhatnagar et al., (2014), who reported that this attributed to the effector inhibition of pesticides on acetylcholinesterase enzyme by pesticides, causing a cholinergic toxidrome excess involving muscarinic, nicotinic, and central nervous system receptors. Also Laudari et al., (2014), was noticed a high frequency of hypokalemia $(12 / 21,57.14 \%)$ and acidosis $(5 / 21,23.81 \%)$ in patients with prolonged QTc which could be the major risk factors for this hazardous ECG abnormality.

As regarding percentage of pesticide exposure and cholinesterase level in the study group; phosphor dithionate (Malathion) is positive in $(70.0 \%)$ and diethyl para-nitrophenyl phosphate (DPT) (DDT) is positive in (62.0\%), while in control group (Malathion) is positive in (4.0\%) and (DPT) is positive in $(6.0 \%)$. The mechanism by which OP compounds induce cardiotoxicity has not been explained thus far, and it is difficult to identify one mechanism as a cause of cardiac intoxication related to organophosphate Senanayake et al., 
(2014) and Worek, et al., (2014).

Positive correlation in the study group of pesticide exposures with serum creatinine, arterial blood gases, serum potassium, moderate and severe reduction in cholinesterase level, cardiac enzyme, (QTc prolongation, ST elevation and ventricular extrasystole) and its significant These results are in agreement with Karki et al., (2014) and Lyzhnikov, et al., (2014), who reported a significant correlation of prolongation in QTc, moderate and severe (OP) intoxication as was shown to be $20-80 \%$ depending on the type of toxic agent and severity of the poisoning.

\section{CONCLUSION}

1. Cardiovascular impacts are a common manifestation following acute (OP) intoxication as cardiac abnormalities (QTc prolongation and ventricular extrasystole) were significantly and detected in most cases.

2. Significant positive correlation in the study group of pesticide exposures were observed with serum creatinine, $\mathrm{ABG}$, serum potassium, moderate and severe reduction in cholinesterase level, cardiac enzyme, QTc prolongation, ST elevation and ventricular extrasystole especially among farmers with moderate severity and living in rural area.

Due to limited literature on cardiac abnormalities of pesticides compounds, it's recommended that;

1. Early diagnosis and perfect treatments with atropine and oximes can certainly save the lives of the patients.

2. Careful observation of EEG changes in patients exposed to (OP) compounds is obligatory, parallel to the suitable medical treatment as a QTc prolongation and ventricular extrasystole can cause lethal arrhythmia.

3. People who are in touch with pesticides, especially those who spray farmlands and gardens, must be used sufficient protective instruments or do consider safety guidelines.

4. More researchers are needed for future testing of other pesticides compounds and cardiac and other vital organs.

5. More community awareness about the hazards of pesticides.

\section{REFERENCES}

Aardema, H.; Meertens, J.; Ligtenberg, J.; Peters-Polman, O.; Tulleken, J.; Zijlstra, J. (2017): Organophosphorus pesticide poisoning: cases and developments. Neth J Med. 66 (4):149-153.

Abby, S. and Sarah, J. (2012): The Health Effects of Pesticides Used for Mosquito Control Principal. Environ Health Perspect. 105 (10):26-30.

Abdollahi, M.; Jalali, N.; Sabzevari, O.; Hosseini. R. and Ghanea, T. (2014): A retrospective study of poisoning in Tehran. $\mathbf{J}$ Toxicol Clin Toxicol. 35; (4):387-393.

Andersson, K. (2009): Effects of cigarette smoking on learning and retention. Psychopharmacology (Berlin); 41:1-5.

Bazett, H.C. (2009): An analysis of the time relationship of electrocardiograms. Heart.7: 353357.

Bhatnagar, V.K.; Sharma, R.P. and Malviya, A.N. (2012): Effects of pesticidal stress amongst pesticide factory workers in Agra India. Public Health. 94(6):375-78.

Campbel, M.J., and Machin, D. (2009): Statistical inference.In: medical Statistical. A common sense approach, Campbel, M, J; and Machin, D (Ed). John Wiley and Sons Chichester, New York, Brisbane, Toronto, Singapore. P: 69. 
Eddleston M. (2010): Patterns and problems of deliberate selfpoisoning in the developing world. QJM. 93:715-731.

Eriksson, M.; Hardell, L.; Berg, N.; Mollner, T. and Axelson, $O$. (2014): Soft tissue sarcomas and exposure to chemical substances. A case-referent study. $\mathrm{Br} \mathrm{J}$ Ind Med. 38:27-33.

Karki, P.; Ansari, J.; Bhandary, S. and Koirala, S. (2014): Cardiac and electrocardiographic manifestations of acute organophosphate poisoning. Singapore medical journal. 45:385389.

Kiss, Z. and Fazekas, T. (2011): Arrhythmias in organophosphate poisonings. Acta Cardiol. 34:32330. 18.

Lacasana, M.; Lopez, I.; Rodriguez, M.; Aguilar C, Blanco, J. and Perez O, (2010): Association between organophosphate pesticides exposure and thyroid hormones in floriculture workers. Toxicol Appl Pharmacol. 243 (1):19-26.

Laudari, S.; Patowary, B.S.; Sharma, S.K.; Dhungel, S.; Subedi, K. and Bhattacharya. (2014): Cardiovascular Effects of Acute Organophosphate Poisoning. Asia Pac J Med Toxicol. 3:64-67.

Lin, T.J.; Walter, F.G.; Hung, D.Z.; Tsai, J.L.; Hu, S.C. and Chang, J.S. (2012): Epidemiology of organophosphate pesticide poisoning in Taiwan. Clinical Toxicology. 46(9):794-801.

Linden, C.H.; Burns, M.J. and Mycyk, M.B. (2017): Poisoning, drug overdose and envenomation. In: Fauci AS, Braunwald E, Kasper DL, Hauser SL, Longo DL, Jameson JL, et al., editors. Harrison's Principles of Internal
Medicine. 17th ed. New York: McGraw-Hill; 2741-2748.

Ludomirsky, A., Klein, H.O, Sarelli, P.; Becker, B.; Hoffman, S. and Taitelman, U. (2014): QT prolongation and polymorphous. The American journal of cardiology.49 (7):1654-8.

Lyzhnikov, E.A.; Savina, A.S. and Shepelev, V.M. (2014): Pathogenesis of disorders of cardiac rhythm and conductivity in acute organophosphate insecticide poisoning. Kardiologiia. 15:126129.

Megarbane, B. (2014): Toxidromebased Approach to Common Poisonings. Asia Pac J Med Toxicol. 3:2-12.

Milena, K.; Ivan, B.; Dragan, D.; Darija, K.; Tepavcevic, A.; Tanja, J. and Tatjana P. (2015): a casecontrol study, hepatocellular carcinoma, risk factors. Institute of Infectious and Tropical Diseases, Clinical Center of Serbia, Belgrade; GIT. (29), 519-P532.

Miller, M.D.; Crofton, K.M.; Rice, D.C. and Zoeller, R.T. (2016): Thyroid-disrupting chemicals: interpreting upstream biomarkers of adverse outcomes. Environ Health Perspect. 117:1033-1041.

Mucci, L.A.; Kuper, H.E.; Tamimi, R.; Lagiou, P., Spanos, E. and Trichopoulos, D. (2001): Age at menarche and age at menopause in relation to hepatocellular carcinoma in women. $\mathrm{Br} \mathrm{J}$ Obstet Gynecol. 108: 291-294.

Om, P. and Ganda, M.D. (2016): diabetes and cardiovascular disease. Lipid Clinic and Diabetes Center. The Journal of emergency medicine. 13 (1):39-42.

Paul, U.K. and Bhattacharyya, A.K. (2012): ECG manifestations of 
acute organophosphorus poisoning. J Indian Med Assoc; 110:98. 17.

Rafighdoost. A.; Mirhoseini, E.; Pourzand, $H$. Rafighdoust, $H$. (2014): $\quad$ Epidemiology of organophosphate poisoning, and its cardiac and pulmonary effects. Journal of Birjand University of medical science. 13 (4 (29):49-54.

Ramazzini, C. (2012): Statement on the control of pesticides in the European Union: A call for action to protect human health. Am J Ind Med; 52:176-7.

Saadeh, A.; Farsakh, N.A. and Ali, M. (2014): Cardiac manifestations of acute carbamate and organophosphate poisoning. Heart. 77:461-464.

Senanayake, N.; de, Silva, H.J. and Karalliedde, L. (2013): A scale to assess the severity of organophosphorus intoxication: POP scale. Hum Exp Toxicol; 12:297-299.

Tafuri, J. and Roberts, J. (2015): Organophosphate poisoning. Annals of emergency medicine.16 (2):193202.

Taira, K.; Aoyama. Y. and Kawamata, M. (2016): Long QT and ST-T change associated with organophosphate exposure by aerial spray. Environmental Toxicology and Pharmacology. 22 (1):40-5.

Verebey, K.; Buchan, B.J. and Turner, C.C. (1998): "Laboratory testing In a clinical textbook of Active Disorder". Frances, R.J., Miller, S.I. (Eds). 2nd ed. Guilford.
Press. New. York, London; PP. 7788.

Xu, Z.; Li, X.; Su, Q.; Xu, L.; Zhang, P. and Kong, Z. (2013): Effect of Synthetic Pyrethroid Pesticide Exposure During Pregnancy on the Growth and Development of Infants. Asia-Pacific Journal of Public Health; 25:72-79.

Yurumez, Y.; Yavuz, Y.; Saglam, H.; Durukan, P.; Ozkan, S. and Akdur, $\quad$ O. (2015): Electrocardiographic findings of acute organophosphate poisoning. $\mathbf{J}$ Emerg Med. 36:39-42.

Xu, Z.; Li, X.; Su, Q.; Xu, L.; Zhang, P. and Kong, Z. (2013): Effect of Synthetic Pyrethroid Pesticide Exposure During Pregnancy on the Growth and Development of Infants. Asia-Pacific Journal of Public Health; 25:72-79.

Yurumez, Y.; Yavuz, Y.; Saglam, H.; Durukan, P.; Ozkan, S. and Akdur, O. (2015): Electrocardiographic findings of acute organophosphate poisoning. $\mathbf{J}$ Emerg Med. 36:39-42.

Yurumez, Y.; Yavuz, Y.; Saglam, H.; Durukan, P.; Ozkan, S. and Akdur, $\quad$ O. (2015): Electrocardiographic findings of acute organophosphate poisoning. $\mathbf{J}$ Emerg Med. 36:39-42.

Yurumez, Y.; Yavuz, Y.; Saglam, H.; Durukan, P.; Ozkan, S. and Akdur, $\quad 0 . \quad$ (2015): Electrocardiographic findings of acute organophosphate poisoning. J Emerg Med. 36:39-42. 


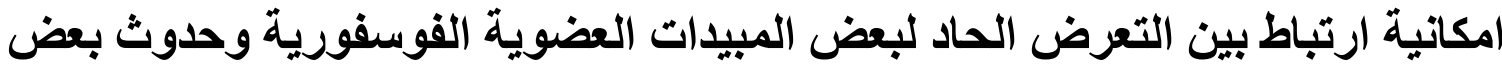 الاضطر ابات بالقلب والتغيرات الإلكيمائية
}

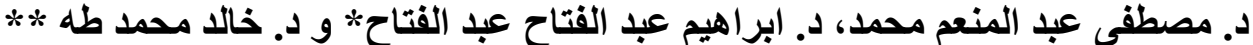

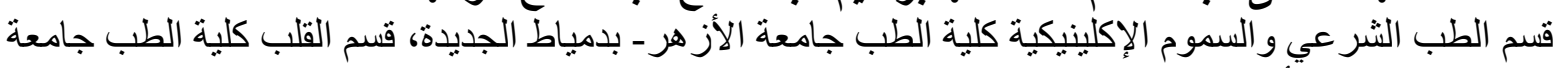

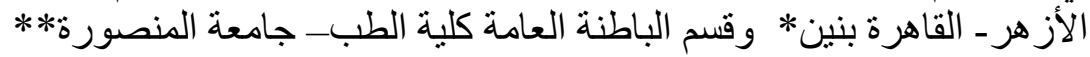

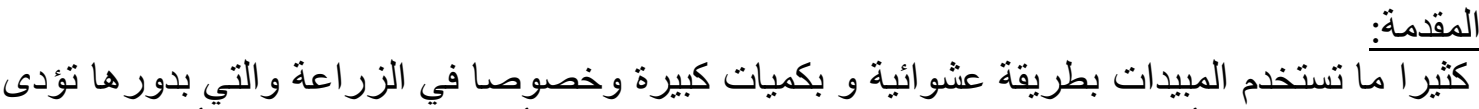

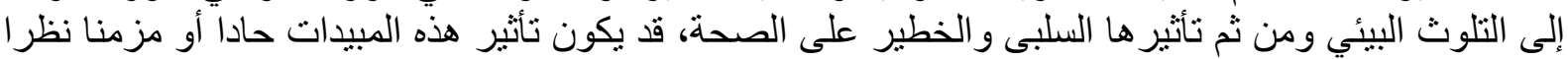

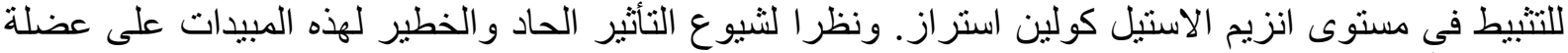

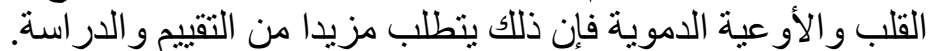
الهو در اسة العلاقة بين إمكانة

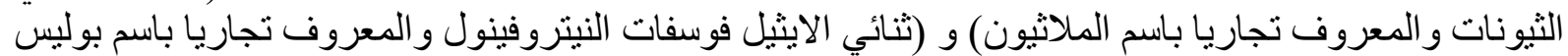

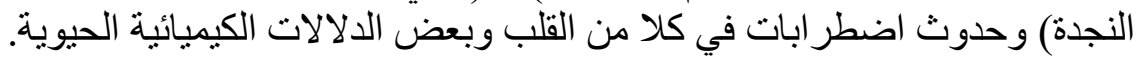

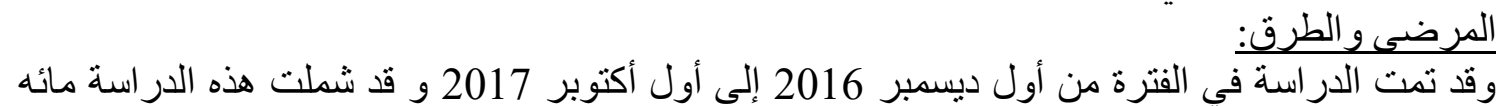
حالة من مستشفى الطوارئ بالمنصورة وقسم الطو ارئ بكلية طب الأز هر بدمياط، كما تم اختيار خمسون شخصا

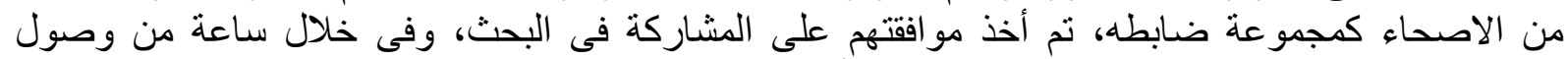

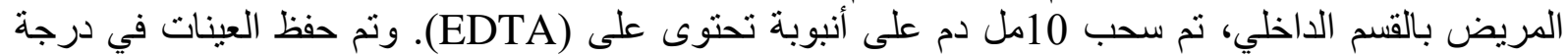

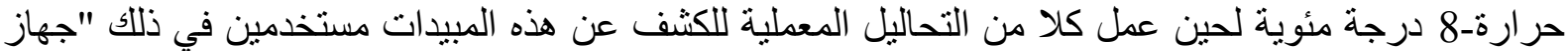

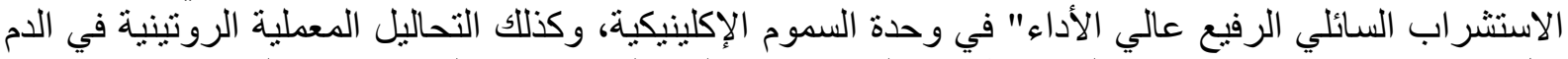

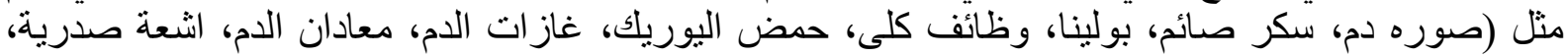

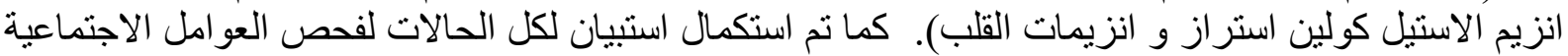

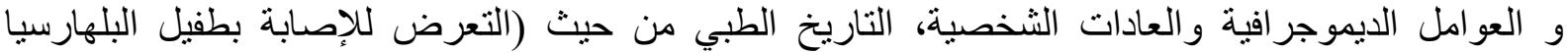

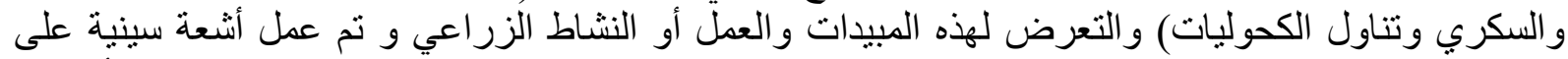

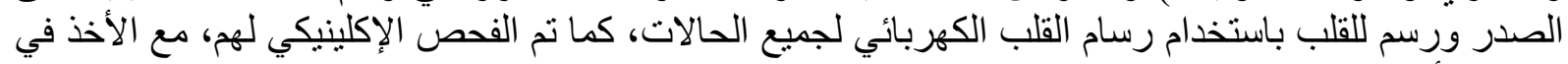
الاعتبار أعر اض الاعتلال القلبي.

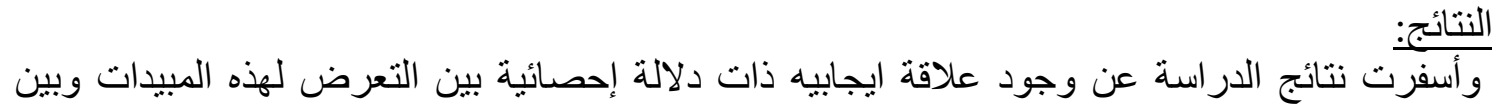

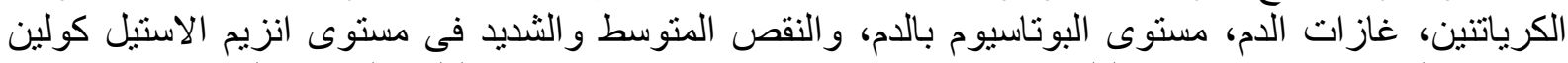

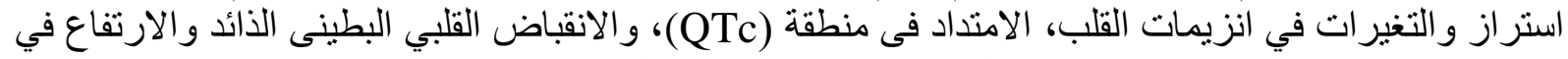

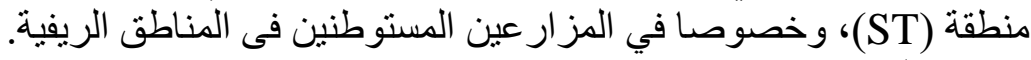
وبناءا على ما سبق نوصن

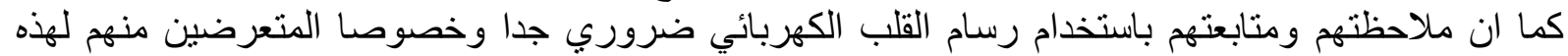

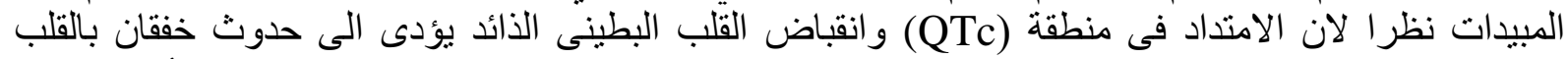

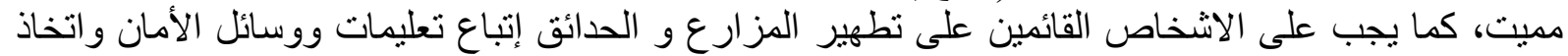

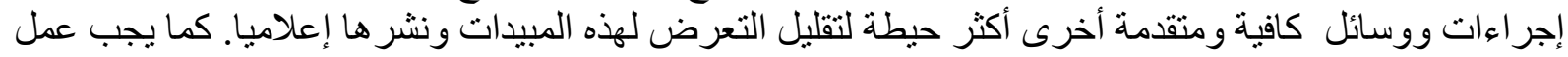

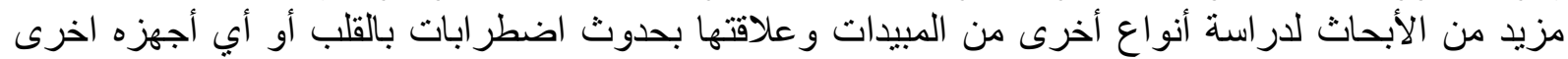

